\title{
Application of the GDDSYN Method in the Era of KEPLER, CoRoT, MOST and BRITE
}

\author{
Stefan W. Mochnacki \\ Dept. of Astronomy \& Astrophysics, University of Toronto, \\ 50 St. George St. Rm. 101, Toronto ON, Canada M5S 3H4 \\ email: stefan@astro.utoronto.ca
}

\begin{abstract}
The precision of observations using observatories in space exceeds by a factor of 100 the accuracy of the light curve and line profile synthesis methods developed decades ago. Furthermore, physical effects too small to detect using ground based observations, such as aberration and Doppler beaming, become important when observing from space.

The GDDSYN method, developed by Hendry and Mochnacki, is both accurate and efficient, and is useful in the new context of space-based observations. Using a geodesic distribution of triangular surface elements varying little in size, it provides an alternative to the WilsonDevinney code used at the heart of PHOEBE, and is adaptable to the new physical effects which are now observable. Tests and improvements are discussed.
\end{abstract}

Keywords. stars: binaries: eclipsing, methods: numerical

\section{Introduction}

It is 43 years since the first synthesis of eclipsing binary light curves based on the Roche model (Lucy 1968), although the first detailed fits to actual observations had to wait for Wilson \& Devinney (1971) and Mochnacki \& Doughty (1972), Lucy (1973) and soon many others, as cited in the comprehensive textbook by Kallrath \& Milone (1998), p.17 ff. Just as the earliest papers were being published, a remarkable conference, IAU Colloquium 16, "Analytical Procedures for Eclipsing Binary Light Curves", was held in Philadelphia, September 8-11, 1971. It was a confrontation between the old analytical methods using simplified geometrical models and the new synthesis computer codes using realistic equipotentials and astrophysics to model close binary stars. I recall that at lunch one day a number of us young synthesizers were sitting with John Merrill, who had devoted much of his life to producing the tables for using the RussellMerrill method (Merrill 1950 and Russell \& Merrill 1952). At some point a visibly energized Dr. Merrill admonished us all:

\section{"You young guys should pay attention to the Tables!"}

It had become clear to him that what us "young guys" had done was to make his grand tool for solving eclipsing binary light curves essentially irrelevant, that the elegance of analytical functions and the vast effort of calculation by hand was supplanted by brute force calculations taking a few minutes on a room-sized mainframe computer. This moment, and everything else at the conference, were crystallized for me later when I read Kuhn's "Structure of Scientific Revolutions" (Kuhn 1962). In a small specialized community, we had experienced a "paradigm shift", with many of the sociological phenomena described by Kuhn. In fact, this advance could have taken place almost a decade earlier, since Lucy's computations were done in 1964 (Moyd 1971) and sufficient computing power was available even earlier. Most of the people attempting the new approach were outsiders to the field, or felt that way. The breakthrough had not come from the centres of eclipsing binary research of the time, and it was somewhat painful for those who had invested so much in the analytical methods.

The advent of "second-generation" computers in the early 1960's allowed realistic physics to be accurately modelled, without needing to simplify to allow analytical solutions. This also 
allowed sophisticated fitting methods to be developed, so that the parameters of the model, or elements of the binary, could be determined with an estimate of their accuracy (e.g. see Chapter 4 of Kallrath \& Milone (1998) and Prsa \& Zwitter (2005) for reviews). The accuracy of the light curves produced by the synthesis codes needed only to to be somewhat better than the photo-electric observations, which had standard deviations typically between 0.003 and 0.01 magnitudes. Although the advent of charge-coupled devices as detectors in the 1980's improved the quality, consistency and quantity of data, errors of less than a millimagnitude or two have rarely if ever been produced from ground-based observations, and therefore the codes developed around 1970 have served admirably, with appropriate refinement (e.g. Wilson 1994).

Two developments in particular have demonstrated the need for improvement of the binary synthesis codes. The first was the application of methods such as Maximum Entropy to determine spot distributions over close binaries (e.g. Maceroni, van't Veer \& Vilhu 1991). This required that a surface element distribution be chosen such that elements did not vary excessively in size, and allowed the determination of the light curve of each element. The second was the advent of photometry obtained using observatories in space, such as MOST (Walker et al. 2003), CoRoT (Baglin et al. 2007) and KEPLER (Koch et al. 2010). These observatories can produce photometry accurate to the order of $10^{-5}$, as opposed to the ground-based limit of about $10^{-3}$. This means that photometry in space is about 100 times more accurate than the main synthesis codes in use since 1970. Not only must the methods be made more precise, but many kinds of hitherto ignored physical effects must be considered to improve accuracy. A "paradigm shift" is once more taking place, and Merrill's words are poignantly apropos, this time for those of us who developed the first generation of synthesis codes.

\section{Accuracy of binary star codes}

The accuracy of binary star light curve and line profile synthesis codes is affected by (a) geometrical factors (including gravity), (b) stellar atmosphere and interior physics, and (c) computational precision (algorithms). The "local" physical issues have been well covered by others at this Symposium and here I will concentrate on (a) and (c), for binaries without accretion disks and other complications beyond their photospheres.

\subsection{Geometrical Issues}

(A) Roche model validity. The traditional Roche model assumes that each component star's mass acts as a point at its center, that there is fully synchronous rotation and that the orbit is circular. Martin (1970) showed that the central point mass assumption was valid to about $1 \%$ in radius, while Limber (1963) and Wilson (1979) showed how to modify the Roche potential for non-synchronous rotation and orbital eccentricity, respectively. However, all of these approaches make simplifying assumptions which should be re-examined in the new accuracy regime.

(B) Relativity. Recently, so-called Doppler boosting has been successfully used to derive radial velocity curves from KEPLER photometry (van Kerkwijk et al. 2010 and references therein). This is caused by the aberration due to redshift, and therefore not only are fluxes and apparent temperatures affected, but also angular sizes. In contact binaries, radial velocity variations of $300-400 \mathrm{~km} / \mathrm{s}$ are observed, which creates effects of order 0.003 magnitudes, far above the spacedbased limit of detection. The whole appearance of the binary is distorted, so that the simple point-source model used by van Kerkwijk et al. (2010) is inadequate for contact or near-contact systems. The Roche model needs to be transformed into special relativity, perhaps by applying the special relativistic theory of Penrose (1959) or general relativity as applied by Kopeikin \& Ozernoy (1999), to obtain the image of the system as it would be seen by an observer with infinite angular resolution. This includes light time effects, gravitational self-lensing (e.g. Rahvar et al. 2011) and gravitational redshift. 


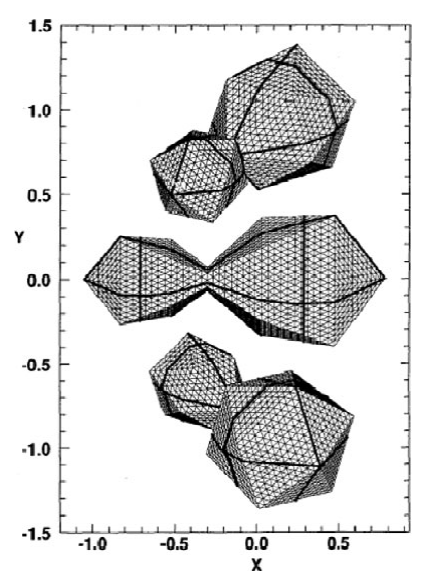

Figure 1. Stretched icosahedron basis, with icosa-faces subtriangulated. Radii are produced from the center of each component though each triangular vertex until they meet the equipotential surface, seen in Fig.2. From Hendry \& Mochnacki (1992).

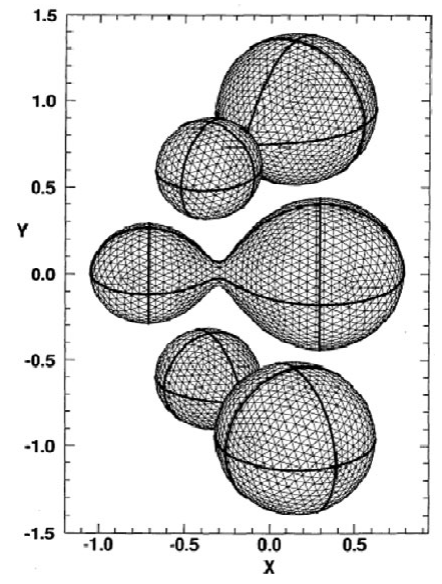

Figure 2. The Roche equipotential surface generated from Fig. 1. This model has mass ratio $\mathrm{q}=0.41$, fill-out $\mathrm{F}=1.1$ and inclination $\mathrm{i}=67$ degrees. Note that detached, semidetached and over-contact configurations can all modelled by GDDSYN. From Hendry \& Mochnacki (1992).

\subsection{Astrophysical Issues}

These include limb darkening (see discussions by Prsa, Nielsen and others in this volume), gravity "darkening" and convection effects, computation of intensities and intensity line profiles, irradiation effects, star spots, and that unsolved mystery, the O'Connell effect.

\section{The GDDSYN Method and Computational Precision}

The GDDSYN method was devised by Hendry \& Mochnacki (1992) to compute the "kernel" for Maximum Entropy Method (MEM) fitting of starspots on close binary systems (Hendry et al. 1992). Later, a method was developed to simultaneously fit the elements and spots of contact binaries using contemporaneous photometric and spectroscopic observations (Hendry \& Mochnacki 2000). Neither the author's GENSYN method (Mochnacki \& Doughty 1972) nor the WD-LC method (Wilson \& Devinney 1971) was used due to numerical inaccuracy and inappropriate pixel geometry.

The GDDSYN code uses a geodesic distribution of elements, determined by producing a radius vector from the center of mass of each component through the sub-vertices on stretched icosahedrons out to the Roche equipotential (Figure 1).

This distribution of elements has many good features, especially because the areas of the elements range by at most a factor of about two, which is very well suited for techniques such as MEM. Ordering of vertices in triangles is counter-clockwise; the limb of an eclipsing component is defined as a convex polygon with about 50-150 vertices projected counter-clockwise on the sky, which allows for easy and rapid determination of the visibility of all elements, including those partially obscured by the limb (Fig. 3).

The triangularity of all elements also means that all elements are always convex polygons, making visibility computation very stable. Thus the light curve of each element can be accurately computed and stored; the set of all element light curves is the "kernel" for spot fitting. As in the Wilson-Devinney and Mochnacki-Doughty methods, recursive irradiation of each element is easily computed, which is not really possible in the original method of Lucy (1968). GDDSYN is faster, more precise and more self-consistent than the Wilson-Devinney code, at least in its older versions (Hendry \& Mochnacki 1992). The inherent smoothness and stability of GDDSYN is 


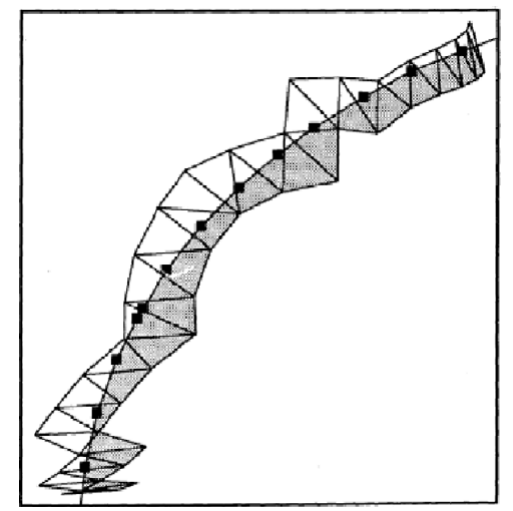

Figure 3. Eclipsing details. The solid squares represent points on a segment of the the limb of the eclipsing component, while the triangles are elements on the eclipsed component. The full limb of the eclipsing component is represented as a convex polygon with order $10^{2}$ points. The intersection of each partially obscured triangular element and the polygonal limb is determined.

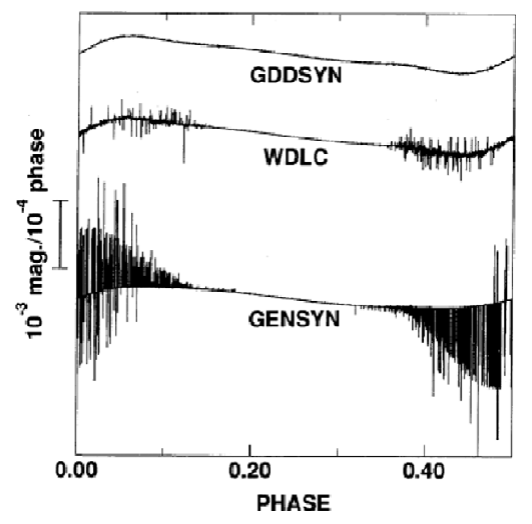

Figure 4. The slopes of light curves evaluated in steps of $10^{-4}$ in phase for a system such as in Fig. 2, comparing GDDSYN with the Mochnacki-Doughty and Wilson-Devinney codes (Hendry \& Mochnacki 1992).

demonstrated in Fig. 4, showing that the basic method has the potential of meeting the precision requirements of space-based photometry. The issue now is whether it is also accurate enough. What are the systematic errors associated with this and other binary synthesis methods?

\section{The Computational Accuracy of Synthesis Techniques}

In Section 2, I discussed fundamental physical issues affecting accuracy. There are also systematic computational issues which affect synthesis codes, since all such codes involve discretization of the radiating surfaces. These are surprisingly large (Hendry \& Mochnacki 1992), and fall into the following classes:

\subsection{Perpendicular to the line of sight}

In GDDSYN, the limb is approximated as a polygon of straight line segments rather than as a curve. Hendry \& Mochnacki (1992) showed that the fractional underestimate of the projected area of the eclipsing component is,

$$
\Delta A_{G D D_{1}}=1-\frac{n}{2 \pi} \sin \frac{2 \pi}{n} \approx \frac{2 \pi^{2}}{3 n^{2}}+\mathcal{O}\left(n^{-4}\right)
$$

where $n$ is the number of line segments defining the outline of the eclipsing component.

The simplest way to reduce this error is to increase the number of points defining the limb where eclipsing takes place, using polynomial interpolation.

\subsection{Along the line of sight}

A problem common with other codes is that along the line of sight, the limb is defined by the first invisible point or the last visible. This means that such a point is not at the true horizon (or limb) as seen by the observer, but $-\theta$ to $+\theta$ from it, where $\theta$ is the mean angular distance between points on the photosphere as seen from the star's centre. Thus the projected radius at the limb will be less, and the eclipsing star's area is underestimated. In the case of GDDSYN, Hendry \& Mochnacki (1992) showed that this error is,

$$
\Delta A_{G D D_{2}} \approx \frac{\pi^{2}}{3 n^{2}}+\mathcal{O}\left(n^{-4}\right)
$$


Hendry \& Mochnacki (1992) for the Wilson-Devinney method estimated an error of,

$$
\Delta A_{W D} \approx \frac{4 \pi^{2}}{3 n^{2}}+\mathcal{O}\left(n^{-4}\right)
$$

where $n=\frac{2 \pi}{\theta}$ and $\theta$ is the mean angular distance between the centres of surface elements.

With $n$ in the range of 50-70, the total error (eqns. 4.1 plus 4.2 ) in the estimate of the projected area of the eclipsing component is about 0.002-0.004 magnitudes.

Recently, I have corrected the "horizon problem" by employing a routine devised by Mochnacki \& Doughty (1972) to compute eclipse contact angles and the projected outline of a binary, but not used in the synthesis code. This routine, SBOUND, is described in Appendix 2 of that paper, and uses the Newton-Raphson technique to solve for two unknowns. In GDDSYN, it is now used to refine the limb, so that the points defining the limb are now much closer to the true horizon and not just the projection of surface elements which happen to be closest to the horizon. The difference between corrected and uncorrected models is shown in Fig. 5 (for the configuration in Fig. 2), and and is below the estimated maximum error above. For a totally eclipsing system, the error is larger, but does not exceed the estimated maximum error.

\subsection{Surface element areas}

The triangular surface elements of GDDSYN can be relatively large and still produce good computational precision (the tests presented here use only 3660 elements). However, if only flat element areas are considered, the surface area of each component is underestimated by about $0.3 \%$ compared with the accurate calculations of Mochnacki (1984). GDDSYN corrects the element areas for curvature, and also corrects the centroid position of each element so it lies on the Roche equipotential above (or below) the centroid of its vertices. Appendix B of Hendry \& Mochnacki (1992) presents the rather messy element area correction function.

\subsection{Future Prospects}

GDDSYN is currently being incorporated into the PHOEBE code (Prsa \& Zwitter 2005), as an alternative to the Wilson-Devinney back-end code PHOEBE currently uses. The aim is to use the atmospheric physics and fitting procedures within Phoebe, but with the faster and more accurate GDDSYN code providing a better tool for fitting models to space-based photometric observations.

\section{Conclusions}

The GDDSYN code was developed originally for maximum entropy method fitting of starspots on contact binaries (Hendry, Mochnacki \& Collier 1992; Hendry \& Mochnacki 2000), but it

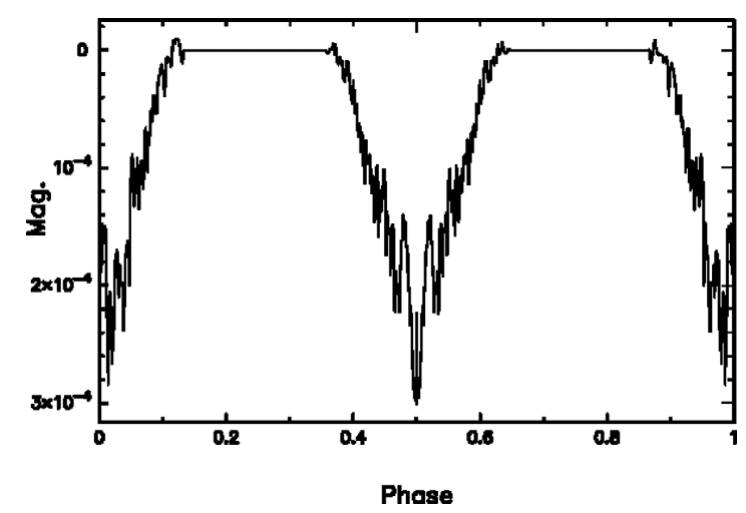

Figure 5. Differences between light curve with horizon refinement and without, for a configuration as in Fig. 2. Refinement increases the projected area of the eclipsing component, hence the minima are deeper. 
now is now highly relevant to the fitting of models to space-based photometric observations and ground-based absorption-cell spectroscopy. The geodesic distribution of triangular surface elements allows computation of light curves and spectral line profiles much more accurately and precisely than older methods based on quadrilateral surface elements. It is being incorporated into PHOEBE.

However, the hundred-fold improvement in photometric precision afforded by space-based observatories such as KEPLER, CoRoT, MOST and others means that not only must the modelling computations be more precise, they also need to be more accurate. This means that special and general relativity must be factored into the model, and that deviations from the classical Roche model must now be considered. This is in addition to the improvement of atmospheric physics, including limb darkening and irradiation, which other authors in this volume are actively working on. There is much important work to be done, and we can truly say that the advent of such precise observations has forced a "paradigm shift" as disturbing as the one around 1971 when synthesis methods replaced the analytical "Royal Road to Eclipses" (Russell 1948).

\section{References}

Baglin, A. et al., 2007, AIP Conf. Proc., 895, 201

Hendry, P. D. \& Mochnacki, S. W. 1992, ApJ, 388, 603

Hendry, P. D. \& Mochnacki, S. W. 2000, ApJ, 531, 467

Hendry, P. D., Mochnacki, S. W., \& Collier, A. C. 1992, ApJ, 399,246

Kallrath, J. \& Milone, E. F. 1998, Eclipsing Binary Stars: Modeling and Analysis (New York: Springer-Verlag)

Koch, D. et al., 2010, ApJ (Letters), 713, L79

Kopeikin, S. M. \& Ozernoy, L. M. 1999, ApJ, 523, 771

Kuhn, T. S. 1962, The Structure of Scientific Revolutions (Chicago: University of Chicago Press) Limber, D. N. 1963, ApJ, 138, 1112

Lucy, L. B. $1968, A p J, 153,877$

Lucy, L. B. 1973, Ap\&SS, 22, 381

Maceroni, C., van't Veer, F., \& Vilhu, O. 1991, ESO Messenger 1991, No. 66, p. 47

Martin, P. G. M. 1970, Ap\&SSS, 7, 119

Merrill, J. E. 1950, Tables for solution of light curves of eclipsing binaries, Contributions from the Princeton University Observatory, no. 23

Mochnacki, S. W. \& Doughty, N. A. 1972, MNRAS, 156, 51

Mochnacki, S. W. 1984, ApJS, 55, 551

Moyd, K. 1971, private communication

Penrose, R. 1959, Mathematical Proceedings of the Cambridge Philosophical Society, 55, 137

Prsa, A. \& Zwitter, T. 2005, ApJ, 628, 426

Rahvar, S., Mehrabi, A., \& Dominik, M. 2011, MNRAS, 410, 912

Russell, H. N. 1948, The Royal Road to Eclipses, in Centennial Papers, Vol. 7 of Harvard College Observatory Monographs, pp. 181-209

Russell, H. N. \& Merrill, J. E. 1952, The Determination of the Elements of Eclipsing Binary Stars, Contributions from the Princeton University Observatory, no. 26, pp. 1-96

van Kerkwijk, M. H., Rappaport, S. A., Breton, R. P., Justham, S., Podsiadlowski, P., \& Han, Z. $2010, A p J, 715,51$

Walker, G. A. H., Matthews, J., Kuschnig, R., Johnson, R., Rucinski, S. M., Pazder, J., Burley, G., Walker, A., Skaret, K., Zee, R., Grocott, S., Carroll, K., Sinclair, P., Sturgeon, H., \& Harron, J. 2003, PASP, 115, 1023

Wilson, R. E. 1979, ApJ, 234, 1054

Wilson, R. E. \& Devinney, E. J. 1971, ApJ, 166, 605 